\title{
A NEW APPROACH TO THE STABILITY PROBLEM OF PLATES SUBJECTED TO ARBITRARY COMPLEX LOADING
}

\author{
DAO HUY BICH
}

Hanoi State University

SUMMARY. The pre-buckling and post-buckling deformation processes are assumed to be less complicated, i.e. processes of average curvature, the influence of complex loading on the stability of plates was analysed in [1]. In this paper eliminating this restriction, postbuckling process may be arbitrary complicated, a generalized expression for determining critical force is formulated by Bubnov-Galiorkin's method and loading parameter method.

\section{PRE-BUCKLING PROCESS}

Let consider a rectangular plate subjected to biaxial compressions of intensities $p(t)$ and $q(t)$. At any moment $t$ there exists a membrane plane stress state in the plate

$$
\sigma_{11}=-p(t), \quad \sigma_{22}=-q(t), \quad \sigma_{12}=\sigma_{33}=\sigma_{32}=\sigma_{31}=0,
$$

so that

$$
\sigma=-\frac{1}{3}(p+q), \quad \sigma_{u} \doteq\left(p^{2}-p q+q^{2}\right)^{1 / 2} .
$$

The strain velocity tensor is determined from the following equations

$$
\begin{aligned}
& \dot{\varepsilon}_{11}=\frac{1}{\sigma_{u} / s}\left(-\dot{p}+\frac{1}{2} \dot{q}\right)-\left(\frac{1}{\phi^{\prime}(s)}-\frac{1}{\sigma_{u} / s}\right) \frac{\left(p \dot{p}+q \dot{q}-\frac{1}{2} p \dot{q}-\frac{1}{2} q \dot{p}\right)\left(p-\frac{1}{2} q\right)}{p^{2}-p q+q^{2}}, \\
& \dot{\varepsilon}_{22}=\frac{1}{\sigma_{u} / s}\left(-\dot{q}+\frac{1}{2} \dot{p}\right)-\left(\frac{1}{\phi \prime(s)}-\frac{1}{\sigma_{u} / s}\right) \frac{\left(p \dot{p}+q \dot{q}-\frac{1}{2} p \dot{q}-\frac{1}{2} q \dot{p}\right)\left(q-\frac{1}{2} p\right)}{p^{2}-p q+q^{2}} .
\end{aligned}
$$

and the arc-length of deformation trajectory is evaluated from

$$
\frac{d s}{d t}=\frac{2}{\sqrt{3}}\left(\dot{\varepsilon}_{11}^{2}+\dot{\varepsilon}_{22}^{2}+\dot{\varepsilon}_{11} \dot{\varepsilon}_{22}\right)^{1 / 2} \equiv F(s, p, q) .
$$

\section{POST-BUCKLING PROCESS}

Suppose that external forces depend on a loading parameter $t$. The parameter $t$ increases and will reach some value $t_{k}$. At this moment $t_{k}$ a bifurcation of equilibrium states is assumed to appear: with an infinitesimal small increment of external force the plate is buckled and receives possible increments of deformation

$$
\delta \varepsilon_{i j}=\delta \varepsilon_{i j}^{*}-Z \delta \chi_{i j}
$$

where 


$$
\delta \chi_{i j}=\frac{\partial^{2} \delta w}{\partial x_{i} \partial x_{j}} \equiv \delta w_{, i j} .
$$

These post-buckling deformation processes may be arbitrary complicated, so that the stressstrain relations are defined by the elastoplastic deformation process theory [2]

$$
\delta \sigma_{i j}=\frac{2}{3} A\left(\delta \varepsilon_{i j}+\delta_{i j} \delta \varepsilon_{k k}\right)+(P-A) \frac{\sigma_{k \ell} \delta \varepsilon_{k \ell}}{\sigma_{u}^{2}} \sigma_{i j}
$$

where

$$
\begin{aligned}
& A=-\frac{\sigma_{u} f}{\sin \theta}=\frac{\sigma_{u}}{s}\left[1+\left(\frac{3 G s}{\sigma_{u}}-1\right) \frac{1-\cos \theta}{2}\right]=\frac{1}{2}\left(3 G+\frac{\sigma_{u}}{s}-\frac{1}{2}\right)\left(3 G-\frac{\sigma_{u}}{s}\right) \cos \theta \\
& P=\frac{\psi}{\cos \theta}=\phi^{\prime}(s)-\frac{3 G-\phi \prime}{\cos \theta} \frac{1-\cos \theta}{2}=\frac{1}{2}\left(3 G+\phi^{\prime}\right)-\frac{1}{2} \frac{3 G-\phi \prime}{\cos \theta}
\end{aligned}
$$

with

$$
\cos \theta=\frac{\sigma_{i j} \delta \varepsilon_{i j}}{\sigma_{u} \delta s}, \quad \delta s=\frac{2}{\sqrt{3}}\left(\delta \varepsilon_{11}^{2}+\delta \varepsilon_{22}^{2}+\delta \varepsilon_{11} \delta \varepsilon_{22}+\delta \varepsilon_{12}^{2}\right)^{1 / 2} .
$$

According to Ilyushin's approximate statement $\delta T_{i j}=0$ and not accounting the unloading, we obtain

$$
\delta \varepsilon_{i j}^{*}=0, \quad \cos \theta=-\frac{Z \sigma_{i j} \delta w_{, i j}}{\sigma_{u} \delta s}, \quad \delta s=\frac{2}{\sqrt{3}} Z\left(\delta w_{, 11}^{2}+\delta w_{, 22}^{2}+\delta w_{, 11} \delta w_{, 22}+\delta w_{, 12}^{2}\right)^{1 / 2} .
$$

Hence the quantity $\cos \theta$ does not depend on $Z$, such that $A$ and $P$ do not depend on $Z$ as well. The bending moments are of the form

$$
\delta M_{i j}=\int_{-h / 2}^{h / 2} \delta \sigma_{i j} Z d Z=\frac{h^{3}}{12}\left[-\frac{2}{3} A\left(\delta w_{, i j}+\delta_{i j} \delta w_{, k k}\right)+(A-P) \frac{\sigma_{i j} \sigma_{k \ell}}{\sigma_{u}^{2}} \delta w_{, k \ell}\right] .
$$

Substituting $\delta M_{i j}$ by (2.3), where $A, P$ contain $\delta w$, into the stability equation

$$
\frac{\partial^{2} \delta M_{i j}}{\partial x_{i} \partial x_{j}}+T_{i j} \delta \chi_{i j}=0
$$

we obtain

$$
\begin{aligned}
& {\left[A-\frac{3}{4}(A-P) \frac{p^{2}}{p^{2}-p q+q^{2}}\right] \frac{\partial^{4} \delta w}{\partial x^{4}}+2\left[A-\frac{3}{4}(A-P) \frac{p q}{p^{2}-p q+q^{2}}\right] \frac{\partial^{4} \delta w}{\partial x^{2} \partial y^{2}}+} \\
& +\left[A-\frac{3}{4}(A-P) \frac{q^{2}}{p^{2}-p q+q^{2}}\right] \frac{\partial^{4} \delta w}{\partial y^{4}}+\frac{9 p}{h^{2}} \frac{\partial^{2} \delta w}{\partial x^{2}}+\frac{9 p}{h^{2}} \frac{\partial^{2} \delta w}{\partial y^{2}}+ \\
& +\frac{1}{2} \frac{\partial^{2} A}{\partial x^{2}}\left(2 \frac{\partial^{2} \delta w}{\partial x^{2}}+\frac{\partial^{2} \delta w}{\partial y^{2}}\right)+\frac{1}{2} \frac{\partial^{2} A}{\partial y^{2}}\left(2 \frac{\partial^{2} \delta w}{\partial y^{2}}+\frac{\partial^{2} \delta w}{\partial x^{2}}\right)+\frac{\partial^{2} A}{\partial x \partial y} \frac{\partial^{2} \delta w}{\partial x \partial y}- \\
& -\frac{3}{4} \frac{p \frac{\partial^{2} \delta w}{\partial x^{2}}+q \frac{\partial^{2} \delta w}{\partial y^{2}}}{p^{2}-p q+q^{2}}\left[p\left(\frac{\partial^{2} A}{\partial x^{2}}-\frac{\partial^{2} P}{\partial x^{2}}\right)+q\left(\frac{\partial^{2} A}{\partial y^{2}}-\frac{\partial^{2} P}{\partial y^{2}}\right)\right]=0
\end{aligned}
$$

Satisfying kinematic boundary conditions with edges simply supported we can find a solution of the form 


$$
\delta w=C \sin \frac{\pi x}{a} \sin \frac{\pi y}{b} .
$$

Notice that $\cos \theta$ does not contain $C$, so that $A, P$ also do not contain $C$. Substituting $\delta w$ into the stability equation and applying Bubnov-Galiorkin's method [3]:

$$
\int_{0}^{a} \int_{0}^{b} X \sin \frac{\pi x}{a} \sin \frac{\pi y}{b} d x d y=0
$$

where $X$ is the expression in the left hand side of the stability equation (2.4), give from (2.5) an equation for finding a critical force.

Consider the case of a square plate. In this case

$$
\begin{aligned}
\delta w & =C \sin \frac{\pi x}{a} \sin \frac{\pi y}{a}, \\
\cos \theta & =-\frac{p+q}{\left(p^{2}-p q+q^{2}\right)^{1 / 2} \frac{2}{\sqrt{3}}\left(3+\operatorname{ctg}^{2} \frac{\pi x}{a} \operatorname{ctg}^{2} \frac{\pi y}{a}\right)^{1 / 2}} .
\end{aligned}
$$

According to (2.6), the equation (2.4) reduces to

$$
\begin{aligned}
X & \equiv\left[\frac{\pi^{4}}{a^{4}}\left(4-\frac{3}{4} \frac{(p+q)^{2}}{p^{2}-p q+q^{2}}\right) A+\frac{3 \pi^{4}}{4 a^{4}} \frac{(p+q)^{2}}{p^{2}-p q+q^{2}} P-\frac{9 \pi^{2}}{a^{2} h^{2}}(p+q)+\right. \\
& +\frac{3 \pi^{2}}{4 a^{2}}\left(-2+\frac{p(p+q)}{p^{2}-p q+q^{2}}\right) \frac{\partial^{2} A}{\partial x^{2}}+\frac{3 \pi^{2}}{4 a^{2}}\left(-2+\frac{q(p+q)}{p^{2}-p q+q^{2}}\right) \frac{\partial^{2} A}{\partial y^{2}}-\frac{3 \pi^{2}}{4 a^{2}} \frac{p(p+q)}{p^{2}-p q+q^{2}} \frac{\partial^{2} P}{\partial x^{2}} \\
& \left.-\frac{3 \pi^{2}}{4 a^{2}} \frac{q(p+q)}{p^{2}-p q+q^{2}} \frac{\partial^{2} P}{\partial y^{2}}\right] \sin \frac{\pi x}{a} \sin \frac{\pi y}{a}+\frac{\pi^{2}}{a^{2}} \frac{\partial^{2} A}{\partial x \partial y} \cos \frac{\pi x}{a} \cos \frac{\pi y}{a}=0 .
\end{aligned}
$$

The expressions of $A$ and $P$ from (2.2) and (2.7) are written in the form

$$
A=\mathcal{A}_{1}(s, p, q)+\mathcal{A}_{2}(s, q, p) f(x, y) \text {. }
$$

where

$$
\begin{aligned}
A_{1} & =\frac{1}{2}\left(3 G+\frac{\sigma_{u}}{s}\right), \quad A_{2}=\frac{1}{2}\left(3 G-\frac{\sigma_{u}}{s}\right) \frac{p+q}{\sigma_{u}} \\
\sigma_{u} & =\left(p^{2}-p q+q^{2}\right)^{1 / 2}, \quad f(x, y)=\frac{\sqrt{3}}{2}\left(3+\operatorname{ctg}^{2} \frac{\pi x}{a} \operatorname{ctg}^{2} \frac{\pi y}{a}\right)^{-1 / 2}
\end{aligned}
$$

and

$$
P=P_{1}(s, p, q)+P_{2}(s, p, q) g(x, y)
$$

where

$$
P_{1}=\frac{1}{2}\left(3 G+\phi^{\prime}\right), \quad P_{2}=\frac{1}{2}\left(3 G-\phi^{\prime}\right) \frac{\sigma_{u}}{p+q}, \quad g(x, y)=\frac{2}{\sqrt{3}}\left(3+\operatorname{ctg}^{2} \frac{\pi x}{a} \operatorname{ctg}^{2} \frac{\pi y}{a}\right)^{1 / 2}=\frac{1}{f(x, y)}
$$

Hence

$$
\frac{\partial^{2} A}{\partial x^{2}}=A_{2} f_{x x}^{\prime \prime}, \quad \frac{\partial^{2} A}{\partial y^{2}}=A_{2} f_{y y}^{\prime \prime}, \quad \frac{\partial^{2} A}{\partial x \partial y}=A_{2} f_{x y}^{\prime \prime}, \quad \frac{\partial^{2} P}{\partial x^{2}}=P_{2} g_{x x}^{\prime \prime}, \quad \frac{\partial^{2} P}{\partial y^{2}}=P_{2} g_{y y}^{\prime \prime} .
$$


Applying Bubnov-Galiorkin's method

$$
\int_{0}^{a} \int_{0}^{a} X \sin \frac{\pi x}{a} \sin \frac{\pi y}{a} d x d y=0,
$$

we obtain

$$
\begin{aligned}
& \frac{\pi^{2}}{4}\left(4-\frac{3}{4} \frac{(p+q)^{2}}{p^{2}-p q+q^{2}}\right) \mathcal{A}_{1}+\frac{3 \pi^{2}}{16} \frac{(p+q)^{2}}{p^{2}-p q+q^{2}} P_{1}+\frac{\pi^{2}}{a^{2}}\left(4-\frac{3}{4} \frac{(p+q)^{2}}{p^{2}-p q+q^{2}}\right) C_{1} \mathcal{A}_{2}+ \\
& +\frac{3 \pi^{2}}{4 a^{2}} \frac{(p+q)^{2}}{p^{2}-p q+q^{2}} C_{5} \mathcal{P}_{2}-\frac{9(p+q)}{4} \frac{a^{2}}{h^{2}}+C_{4} \mathcal{A}_{2}+\frac{3}{4}\left(-2+\frac{p(p+q)}{p^{2}-p q+q^{2}}\right) C_{2} \mathcal{A}_{2}+ \\
& +\frac{3}{4}\left(-2+\frac{q(p+q)}{p^{2}-p q+q^{2}}\right) C_{3} \mathcal{A}_{2}-\frac{3}{4} \frac{p(p+q)}{p^{2}-p q+q^{2}} C_{6} P_{2}-\frac{3}{4} \frac{q(p+q)}{p^{2}-p q+q^{2}} C_{7} P_{2}=0 .
\end{aligned}
$$

where $C_{i}(i=1 \div 7)$ are constants, evaluated by the following integrals

$$
\begin{aligned}
& C_{1}=\int_{0}^{a} \int_{0}^{a} f(x, y) \sin ^{2} \frac{\pi x}{a} \sin ^{2} \frac{\pi y}{a} d x d y ; \quad C_{2}=\int_{0}^{a} \int_{0}^{a} f_{x x}^{\prime \prime} \sin ^{2} \frac{\pi x}{a} \sin ^{2} \frac{\pi y}{a} d x d y ; \\
& C_{3}=\int_{0}^{a} \int_{0}^{a} f_{y y}^{\prime \prime} \sin ^{2} \frac{\pi x}{a} \sin ^{2} \frac{\pi y}{a} d x d y ; \quad C_{4}=\int_{0}^{a} \int_{0}^{a} f_{x y}^{\prime \prime} \sin \frac{\pi x}{a} \cos \frac{\pi x}{a} \sin \frac{\pi y}{a} \cos \frac{\pi y}{a} d x d y ; \\
& C_{5}=\int_{0}^{a} \int_{0}^{a} g(x, y) \sin ^{2} \frac{\pi x}{a} \sin ^{2} \frac{\pi y}{a} d x d y ; \\
& C_{6}=\int_{0}^{a} \int_{0}^{a} g_{x x}^{\prime \prime} \sin ^{2} \frac{\pi x}{a} \sin ^{2} \frac{\pi y}{a} d x d y ; \quad C_{7}=\int_{0}^{a} \int_{0}^{a} g_{y y}^{\prime \prime} \sin ^{2} \frac{\pi x}{a} \sin ^{2} \frac{\pi y}{a} d x d y .
\end{aligned}
$$

Substituting the expressions of $\mathcal{A}_{1}, \mathcal{A}_{2}, P_{1}, P_{2}$ into the equation (2.11), from which we get the formula for determining a critical force

$$
\begin{aligned}
i^{2} & \equiv \frac{9 a^{2}}{h^{2}}=\frac{4}{p+q}\left\{\frac{\pi^{2}}{2}\left(3 G+\frac{\sigma_{u}}{s}\right)+\frac{3 \pi^{2}}{32}\left(\phi^{\prime}-\frac{\sigma_{u}}{s}\right) \frac{(p+q)^{2}}{p^{2}-p q+q^{2}}+\right. \\
& +\frac{1}{2}\left(3 G-\frac{\sigma_{u}}{s}\right) \frac{p+q}{\sigma_{u}}\left[\frac{\pi^{2}}{a^{2}}\left(4-\frac{3}{4} \frac{(p+q)^{2}}{p^{2}-p q+q^{2}}\right) C_{1}+\frac{3}{4}\left(-2+\frac{p(p+q)}{p^{2}-p q+q^{2}}\right) C_{2}+\right. \\
& \left.+\frac{3}{4}\left(-2+\frac{q(p+q)}{p^{2}-p q+q^{2}}\right) C_{3}+C_{4}\right]+\frac{1}{2}\left(3 G-\phi^{\prime}\right) \frac{\sigma_{u}}{p+q}\left[\frac{3 \pi^{2}}{4 a^{2}} \frac{(p+q)^{2}}{p^{2}-p q+q^{2}} C_{5}-\right. \\
& \left.\left.-\frac{3}{4} \frac{p(p+q)}{p^{2}-p q+q^{2}} C_{6}-\frac{3}{4} \frac{q(p+q)}{p^{2}-p q+q^{2}} C_{7}\right]\right\} \equiv H(s, p, q),
\end{aligned}
$$

where $\sigma_{u}=\left(p^{2}-p q+q^{2}\right)^{1 / 2}$

Now, suppose that $p \equiv p(t), q \equiv q(t)$ are known as functions of loading parameter $t$. The equations (1.3) and (2.12) are satisfied simultaneously, from that we can determine a critical value $t_{k}$ of the loading parameter. Then the critical forces are as follows

$$
p_{t h}=p\left(t_{k}\right) ; \quad q_{t h}=q\left(t_{k}\right) .
$$


In particular case when post-buckling process is a process of average curvature, we have

$$
A=\frac{\sigma_{u}}{s}, \quad P=\phi^{\prime}(s)
$$

from (2.2) to get

$$
\cos \theta=-\frac{p+q}{\sigma_{u}} f(x, y)=1
$$

the functions $f(x, y)$ and $g(x, y)$ must be constant. Hence we obtain

$$
-\frac{p+q}{\sigma_{u}} C_{1}=\frac{a^{2}}{4}, \quad-\frac{\sigma_{u}}{p+q} C_{5}=\frac{a^{2}}{4}, \quad C_{2}=C_{3}=C_{4}=C_{6}=C_{7}=0 .
$$

Finally, from (2.12) we get the known expression of the critical force in [1]

$$
i^{2} \equiv \frac{9 a^{2}}{h^{2}}=\frac{4}{p+q}\left[\pi^{2} \frac{\sigma_{u}}{s}+\frac{3 \pi^{2}}{16}\left(\phi^{\prime}-\frac{\sigma_{u}}{s}\right) \frac{(p+q)^{2}}{p^{2}-p q+q^{2}}\right]
$$

or

$$
i^{2} \equiv \frac{9 a^{2}}{h^{2}}=\frac{3 G \pi^{2} \varphi_{N}}{p+q}\left[4-\frac{3}{4}\left(1-\frac{\varphi_{t}}{\varphi_{N}}\right) \frac{(p+q)^{2}}{p^{2}-p q+q^{2}}\right]
$$

where

$$
\varphi_{N}=\frac{\sigma_{u} / s}{3 G}=\frac{\left(p^{2}-p q+q^{2}\right)^{1 / 2}}{3 G s}, \quad \varphi_{t}=\frac{\phi^{\prime}(s)}{3 G}
$$

\section{CONCLUSIONS}

1. A typical example on the application of the general elastoplastic deformation process theory in the stability problem of plates is given.

2. Establishing a method for formulating an expression of critical force in the general case without any restriction on the arbitrary complex loading, this approach has a practical meaning in engineering calculation.

3. Received result in [1] is a particular case of the generalized expression (2.12) shown in this paper.

\section{REFERENCES}

1. Dao Huy Bich. Influence of complex loading on the stability outside elastic limit of thin plates. Journal of Mechanics (Tap chi co hoc), V. 11, No 3, 1989, pp. 1-7.

2. Dao Huy Bich. Research of the local theory of elastoplastic deformation processes. Dr. Sc. Thesis, The State University of Moscow, 1988 (in Russian).

3. Volmir A. S. Stability of deformable Systems, Edition of Sciences, Moscow, 1967 (in Russian).

Received January 11, 1992

\section{MộT CÁCH TIẾP CẬN MỚI BẢI TOÁN ỔN ĐỊNH CƯA BẢN CHỊU TẢI PHỨC TẠP BẤT KỲ}

Gỡ bỏ mọi giả thiết hạn chế lên quá trình biến dạng sau khi mất ổn định, ở đây có thể xem đó là quá trình phức tạp bất kỳ. Bằng phương pháp Bubnov - Galiorkin và phương pháp tham số tải đã thiết lập được công thức hiển xác định lực tới hạn tổng quát hơn kết quả nhận được trước dây. 